\title{
Characterization of the oxide films formed at the surface of Ni-base alloys in pressurized water reactors primary coolant by transmission electron microscopy
}

Mohamed Sennour $^{1, a}$, Loïc Marchetti
Michèle Pijolat
Mie, , Olivier Raquet

${ }^{1}$ Ecole des mines de Paris, ParisTech - Centre des Matériaux- UMR CNRS 7633 - BP 87- 91003 Evry, France

${ }^{2}$ CEA-DEN/DANS/DPC/SCCME/Laboratoire d'Etude de la Corrosion Aqueuse, CEA
Saclay - Bâtiment $458-91191$ Gif sur Yvette Cédex, France

${ }^{3}$ LPMG-UMR CNRS 5148, Centre SPIN, Ecole Nationale Supérieure des Mines 158

Cours Fauriel - 42023 Saint-Etienne, France

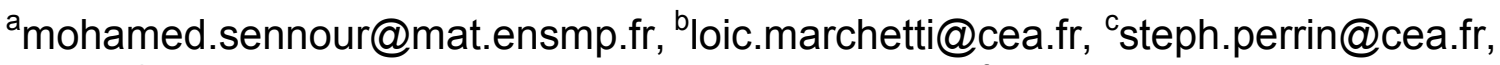
dregine.molins@ensmp.fr, ${ }^{\mathrm{e} m p i j o l a t @ e m s e . f r, ~}{ }^{\mathrm{f}}$ olivier.raquet@cea.fr

Keywords: Corrosion, TEM, Ni-base alloy, primary coolant

Abstract. The oxide film formed on nickel-based alloys in Pressurized Water Reactors (PWR) primary coolant conditions $\left(325^{\circ} \mathrm{C}\right.$, aqueous media) has been investigated by Transmission Electron Microscopy (TEM). TEM observations revealed an oxide layer divided in two parts. The internal layer was mainly composed of a continuous spinel layer, identified as a mixed iron and nickel chromite $\left(\mathrm{Ni}_{(1-\mathrm{x})} \mathrm{Fe}_{\mathrm{x}} \mathrm{Cr}_{2} \mathrm{O}_{4}\right)$. Moreover, nodules of $\mathrm{Cr}_{2} \mathrm{O}_{3}$ were present at the interface between this spinel and the alloy. The external layer is composed of large crystallites corresponding to a spinel structure rich in iron $\left(\mathrm{Ni}_{(1-z)} \mathrm{Fe}_{(2+z)} \mathrm{O}_{4}\right)$ resulting from precipitation phenomena. The influence of alloy surface defects was also studied underlining two main consequences on the formation of the passive film e.g. the internal layer. On one hand, the growth kinetics of the internal spinel rich in chromium increased with the surface defect density. Besides that, when the defect density increased, the oxide scale became more finely crystallized. This result agrees with a growth mechanism due to a rate limiting process of diffusion through the grain boundaries of the oxide. On the other hand, the quantity of $\mathrm{Cr}_{2} \mathrm{O}_{3}$ nodules increased with the number of surface defects, revealing that the nodules nucleated preferentially at defect location.

\section{Introduction}

The corrosion behavior of nickel-base alloys in hig- temperature and high-pressure water has been studied in order to simulate corrosion of steam generators (SG) of pressurized water reactors (PWR) in the primary circuit i.e. in an aqueous medium at a temperature between $285^{\circ} \mathrm{C}-325^{\circ} \mathrm{C}$ and a hydrogen concentration between $25-50 \mathrm{~cm}^{3} \cdot \mathrm{kg}^{-1}$ (NTP). In these conditions, the passivity of these alloys is due to the formation of a protective oxide film which acts as a diffusion barrier and consequently reduces the corrosion rate. In fact, the passive film reduces the release of corrosion products, as nickel cations, in primary water. ${ }^{58} \mathrm{Ni}$ released in the primary circuit is activated into ${ }^{58} \mathrm{Co}$ in the nuclear core under neutronic flux, increasing the global radioactivity of the primary circuit of PWR. For safety reasons for the maintenance staff, the control and restriction of the release of nickel species in the primary circuit is of crucial importance. So the knowledge of the 
nature and the structure of passive films formed on these alloys is essential for a better understanding of the mechanisms governing the phenomena of oxidation and release.

Many published papers describe the oxide film growing on nickel-base alloys as having a duplex structure [1-7]. This duplex oxide has been described as an inner Cr-rich layer and an outer layer consisting of scattered octahedral Fe-rich crystals [2,5] attributed to precipitation phenomena which occurs in saturated media $[3,6]$. The size of these crystallites varies in the range of $100 \mathrm{~nm}$ to $1 \mu \mathrm{m}[5,8-11]$. On the contrary, when corrosion tests were carried out in continuously cleaned and purified media, the crystallite density was drastically decreased [6] in agreement to unsaturated conditions. In these conditions, the duplex morphology has been characterized as an internal Cr-rich layer and a thicker external layer rich in $\mathrm{Cr}$ and $\mathrm{Ni}[4,6]$. If we consider that the duplex structure designation refers to a layer of scattered precipitated crystallites surmounting a continuous and protective oxide film, the question of a multi-layer structure for the protective oxide film can be asked. In this way, Machet [5] suggests a $\mathrm{N}_{\mathrm{Cr}} / \mathrm{N}_{\mathrm{Ni}}$ ratio around 2 in the inner oxide film, consistent with the external layer described by Carrette [4]. In a more recent paper [7], a duplex structure composed by an inner $\mathrm{Cr}_{2} \mathrm{O}_{3}$ layer and an outer $\mathrm{Ni}(\mathrm{OH})_{2}$ layer was suggested after an XPS study. Some authors $[9,11,12]$ also show at the oxide alloy interface a depleted chromium layer in the alloy. Facing these different works, the nature of the passive film formed on nickel base alloys in PWR primary water conditions is not well defined. So, one of the objectives of this work is to obtain a better understanding of the passive film structure by using advanced TEM techniques. Moreover, the surface state of the alloy, such as cold working near surface, plays a key role on the oxidation and release rates $[2,4,9,13]$. As a consequence, the other objective of this study is to understand how the surface defects can modify the passive film.

\section{Experimental}

Materials. Two alloys were used in this work: an Alloy 690 and a Ni-30Cr alloy. The compositions of these alloys are given in Table 1 . Coupons $(30 \times 20 \times 2.5 \mathrm{~mm})$ were mechanically mirrorpolished with $\mathrm{SiC}$ paper up to grade 1200, diamond paste up to $1 \mu \mathrm{m}$ and alumina gel finish. Then samples were cleaned first with distilled water and then with an ethanol-acetone binary mixture in ultrasonic bath.

Table.1 Composition of the alloys (in weight percent)

\begin{tabular}{|c|c|c|c|c|c|c|c|c|c|c|c|c|}
\hline Alloy & $\mathrm{Ni}$ & $\mathrm{Cr}$ & $\mathrm{Fe}$ & $\mathrm{C}$ & $\mathrm{Co}$ & $\mathrm{Mn}$ & $\mathrm{Al}$ & $\mathrm{Ti}$ & $\mathrm{Cu}$ & $\mathrm{Si}$ & $\mathrm{S}$ & $\mathrm{P}$ \\
\hline 690 & 59,31 & 29,20 & 9,94 & 0,018 & 0,014 & 0,31 & 0,13 & 0,27 & $<0,002$ & 0,27 & $<0,0005$ & 0,007 \\
\hline Ni-30Cr & 69,15 & 30,35 & 0,25 & 0,002 & - & 0,02 & 0,06 & 0,04 & - & 0,1 & 0,0009 & - \\
\hline
\end{tabular}

In order to study the influence of surface defect density on the oxide scale morphology while maintaining a polished surface to facilitate the characterization observations, surface of Alloy 690 samples were implanted with Xenon. The coupons were exposed to a beam of $\mathrm{Xe}^{3+}$ ions with an energy of $180 \mathrm{keV}$. The number of implanted ions was about $20.10^{15}$ atoms by $\mathrm{cm}^{2}$ and the affected depth of alloy was about $200 \mathrm{~nm}$. This treatment made it possible to obtain a polished sample with a number of surface defects higher than simply-polished coupons. These implantation defects were intended to simulate a higher cold-worked surface.

Corrosion experiments. The corrosion experiments were performed at $325{ }^{\circ} \mathrm{C}$, under 155 bar in a recirculation autoclave. In this testing device, the pressurized hot water was continuously cleaned 
and purified with the aim of maintaining the concentrations of metallic cations at a low level. Different corrosion durations were investigated between $66 \mathrm{~h}$ and $858 \mathrm{~h}$. The aqueous solution contained $2 \mathrm{mg} . \mathrm{L}^{-1}$ lithium and $1000 \mathrm{mg} . \mathrm{L}^{-1}$ boron. An hydrogen overpressure of 0.29 bar was maintained to ensure a dissolved $\mathrm{H}_{2}$ concentration of $1.310^{-3}$ mol. $\mathrm{L}^{-1}$ and a low oxygen content $\left(\mathrm{O}_{2}\right.$ $<10 \mathrm{ppb}$ ). In these experimental conditions, the calculated $\mathrm{pH}_{325^{\circ} \mathrm{C}}$ of the solution was 7.2.

TEM experiments. Transmission electron microscopy (TEM) characterizations were made on specimen cross-section. Before preparation, the sample was about $1 \mathrm{~cm}$ square. Two bars about 2.5 $\mathrm{mm}$ width were cut along the plane of interest. The two bars were glued together with an epoxy resin, the surface of the oxide layers facing inwards so it was protected from damage, forming a sandwich, which was then cut into slices of $0.5 \mathrm{~mm}$ thickness. A slice was first thinned mechanically to a thickness of 15 to $10 \mu \mathrm{m}$ using a precision tripod device in order to keep the sample absolutely horizontal. The sample was mounted onto a copper grid and thinned to a thickness of a few nanometers from both sides by low-angle $\left( \pm 7^{\circ}\right)$ ion milling with $4-\mathrm{keV}$ argon ions using a Gatan $($ Precision Ion Polishing System (PIPS). Final milling was performed at reduced beam energy $(2 \mathrm{KeV})$ and reduced incident angles $\left( \pm 4^{\circ}\right)$ to remove most of the visible ion damage. TEM investigations were carried out using a Tecnai F20 ST field emission gun microscope, equipped with an Energy Dispersive X-ray (EDX) device, Gatan Imaging Filter (GIF) and Scanning Transmission Electron Microscopy (STEM) system. The latter combined with a High Angle Annular Dark-Field (HAADF) detector allows chemical contrast imaging, using images formed with electrons diffracted at high angle ( $>40 \mathrm{mrad}$ ) where the intensity is sensitive to the atomic number $\mathrm{Z}$ of elements. The spatial resolution of these images depends on the probe size. EDX analysis has been performed in line-scan mode to provide local chemical composition variation over oxide layers. High-resolution transmission electron microscopy (HRTEM) images were obtained using a CCD camera and analyzed by Fourier technique to investigate structural and crystallographic details of the oxide layer.

\section{TEM results}

The use of nanometric-sized probe $(1-2 \mathrm{~nm})$ and high spatial resolution $(\sim 0.24 \mathrm{~nm})$ make possible fine structural and chemical analysis of oxide layers. The following paragraphs present results of TEM, HRTEM, HAADF and EDX investigations carried out on Ni-30Cr and Alloy 690 exposed for different durations in simulated PWR primary water.

Short time experiments. Fig. 1(a) shows a STEM-HAADF image of the oxide film developed on Alloy 690 exposed for 66h. The duplex structure of the oxide layer is readily distinguished. The external oxide scale appeared to be constituted of separated fine crystallites with sizes ranging around $20 \mathrm{~nm}$. Below, a thin continuous and compact layer was observed. The thickness of this layer ranged around $5 \mathrm{~nm}$. Chemical analysis (Fig. 1(b)) revealed an internal oxide layer enriched in chromium and whose composition was consistent with $(\mathrm{Ni}, \mathrm{Fe}) \mathrm{Cr}_{2} \mathrm{O}_{4}$. The external layer was richer in iron $(\sim 17 \mathrm{wt} \%)$ than the internal layer. Fig. 1(c) shows oxide penetration under a continuous internal layer. The EDX analysis performed across this penetration (Fig. 1(d)) indicated that this oxide was enriched in chromium compared to the continuous scale. 

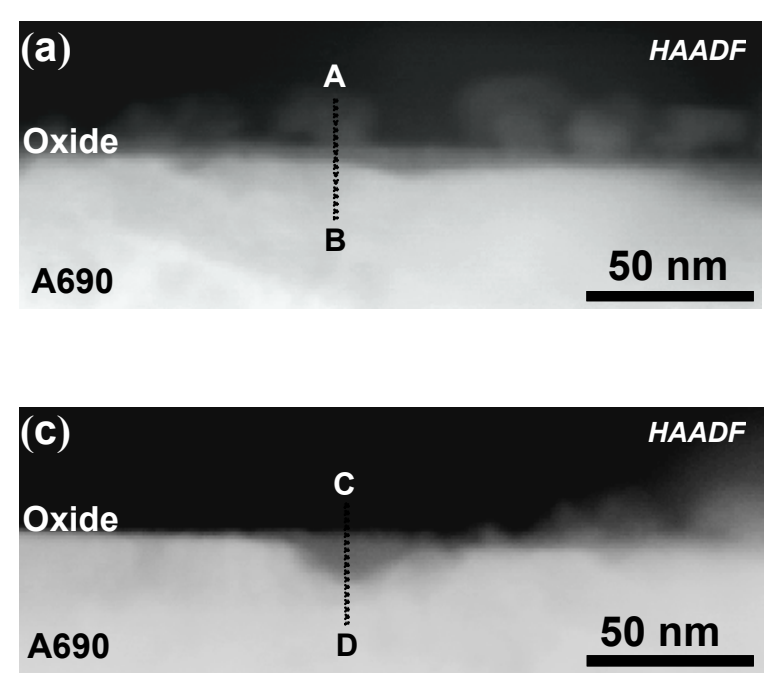

(b)

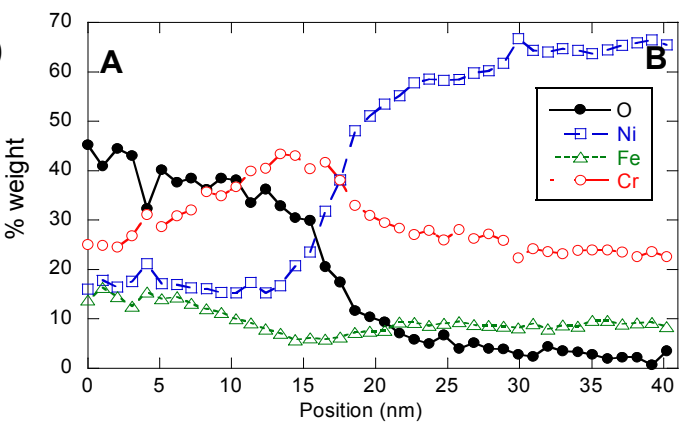

(d)

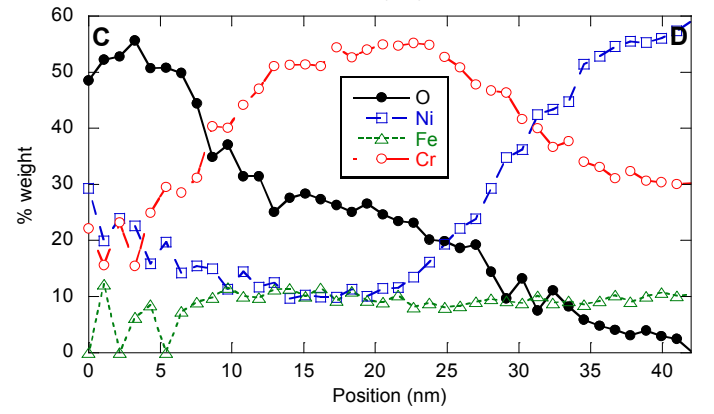

Fig. 1. STEM-HAADF image obtained on the oxide layer developed on Alloy 690 exposed $66 \mathrm{~h}$ in simulated PWR primary water ((a), (c)) and associated chemical analysis profiles ((b), (d)).

HRTEM observations performed on a cross-sectional specimen prepared from the $\mathrm{Ni}-30 \mathrm{Cr}$ alloy exposed 66h in simulated primary water (Fig. 2(a)) revealed small nodules (ranging between 5 and $10 \mathrm{~nm}$ in size) along the oxide/matrix interface. Fourier transform (FT) diffractogram (Fig. 2(b)) identified them as having the $\mathrm{Cr}_{2} \mathrm{O}_{3}$-structure with an orientation relationship with the neighboring matrix of the type: $[2 \overline{2} 01]_{\mathrm{Cr}_{2} \mathrm{O}_{3}} / /[001]_{\text {Matrix }}$ and $(\overline{2} 116)_{\mathrm{Cr}_{2} \mathrm{O}_{3}} / /(200)_{\text {Matrix }}$.

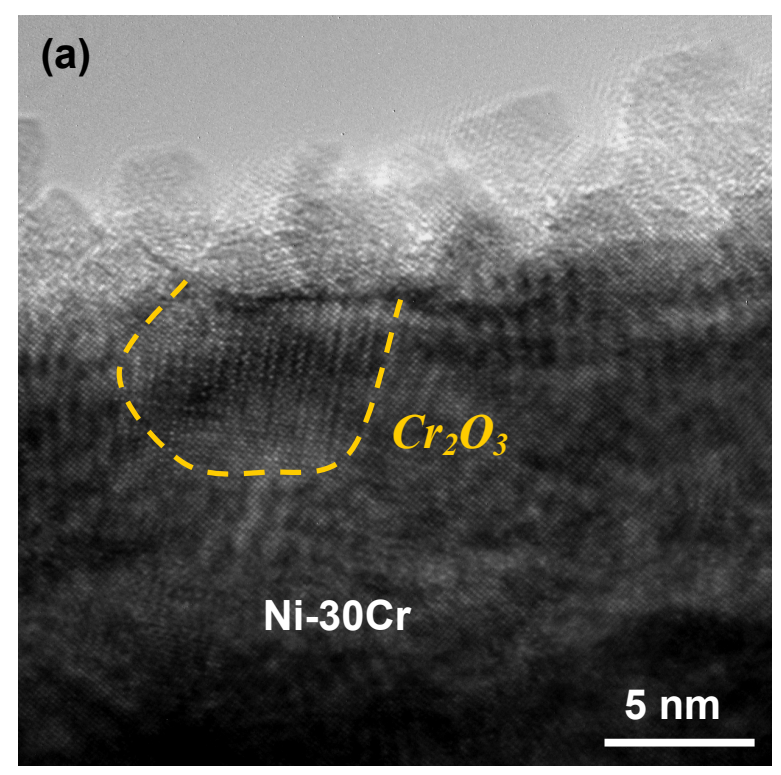

(b)

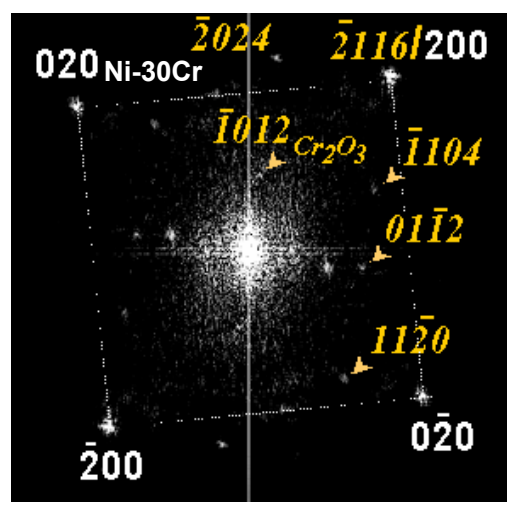

Fig. 2. HRTEM image (a) and FT diffractogram (b) of framed area identified $\mathrm{Cr}_{2} \mathrm{O}_{3}$-structure nodules on Ni-30Cr alloy exposed for $66 \mathrm{~h}$ in simulated PWR primary conditions.

Long time experiments. The oxide scale developed on Alloy690 exposed for $858 \mathrm{~h}$ is shown on Fig. 3(a). The scale had a duplex structure and the external oxide layer consisted of separated 
crystallites which could be up to $100 \mathrm{~nm}$ in size. In contrast to this external oxide, the internal layer appeared to be continuous and compact, with a constant thickness ranging around $50 \mathrm{~nm}$. The HAADF image (Fig. 3(b)) provides a clear indication of the layer thicknesses and morphology. Chemical analysis (Fig. 3(c)), performed on the oxide layer, confirmed the chromium enrichment (43 wt $\%$ ) of the internal layer with elements atomic concentrations in agreement with the $(\mathrm{Ni}, \mathrm{Fe}) \mathrm{Cr}_{2} \mathrm{O}_{4}$ composition. Also as expected, compositional profiles confirmed the iron-enrichment of the large external crystallites in agreement with the $\mathrm{Ni}(\mathrm{Fe}, \mathrm{Cr})_{2} \mathrm{O}_{4}$ composition. Furthermore, EDX analysis performed on the underlying metal did not reveal any $\mathrm{Cr}$-depleted zone below the oxide layer.
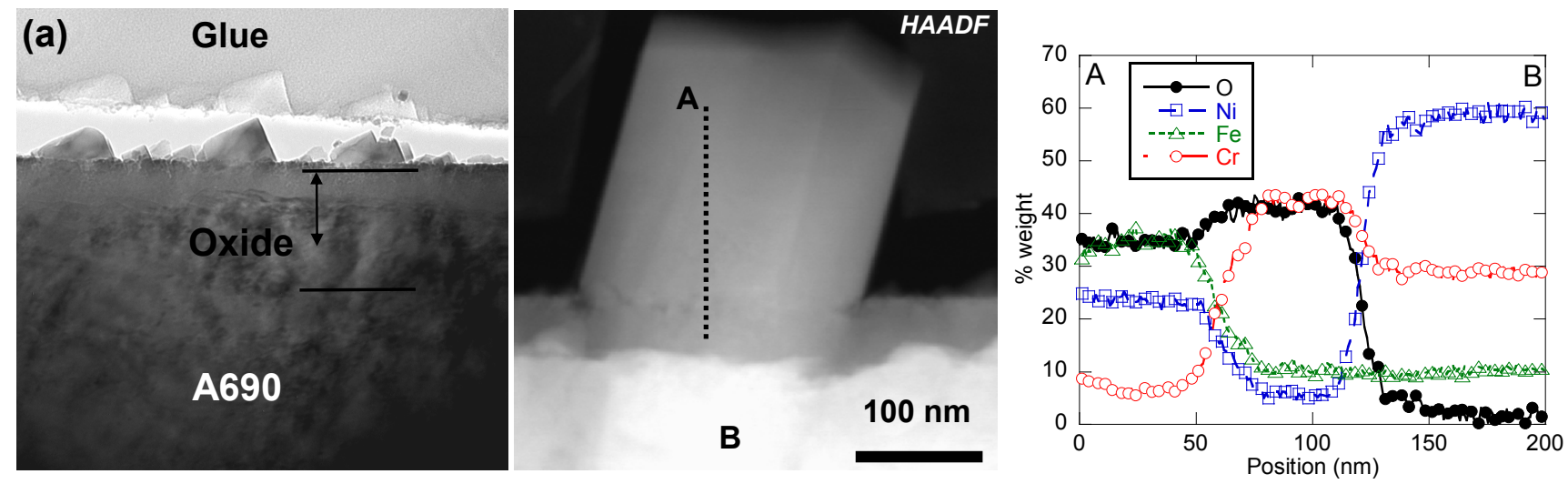

Fig. 3. TEM investigation of Alloy 690 corroded $858 \mathrm{~h}$ in PWR primary conditions (a), TEM/HAADF (b) and associated chemical analyses (c).

Crystallographic investigations were carried out using HRTEM analysis. Fig. 4(a) shows an HRTEM image obtained along the [110] matrix zone axis. The indexing of the corresponding Fourier transform diffractogram (Fig. 4(b)) confirmed the $\mathrm{NiCr}_{2} \mathrm{O}_{4}$-structure of the internal oxide layer with partial substitution of $\mathrm{Cr}$ by $\mathrm{Fe}$ as indicated by EDX analysis (Fig. 3(c)). Furthermore, FT diffractogram analysis revealed parallel orientation with respect to the matrix. HRTEM investigation of the alloy-oxide interface (Fig.4(c)) highlighted the regular presence of small nodules along the interface. These were identified using FT diffractogram analysis (Fig. 4(d)) as having the $\mathrm{Cr}_{2} \mathrm{O}_{3}$-structure, the same as those observed in Ni-30Cr alloy after the short-time corrosion test. 


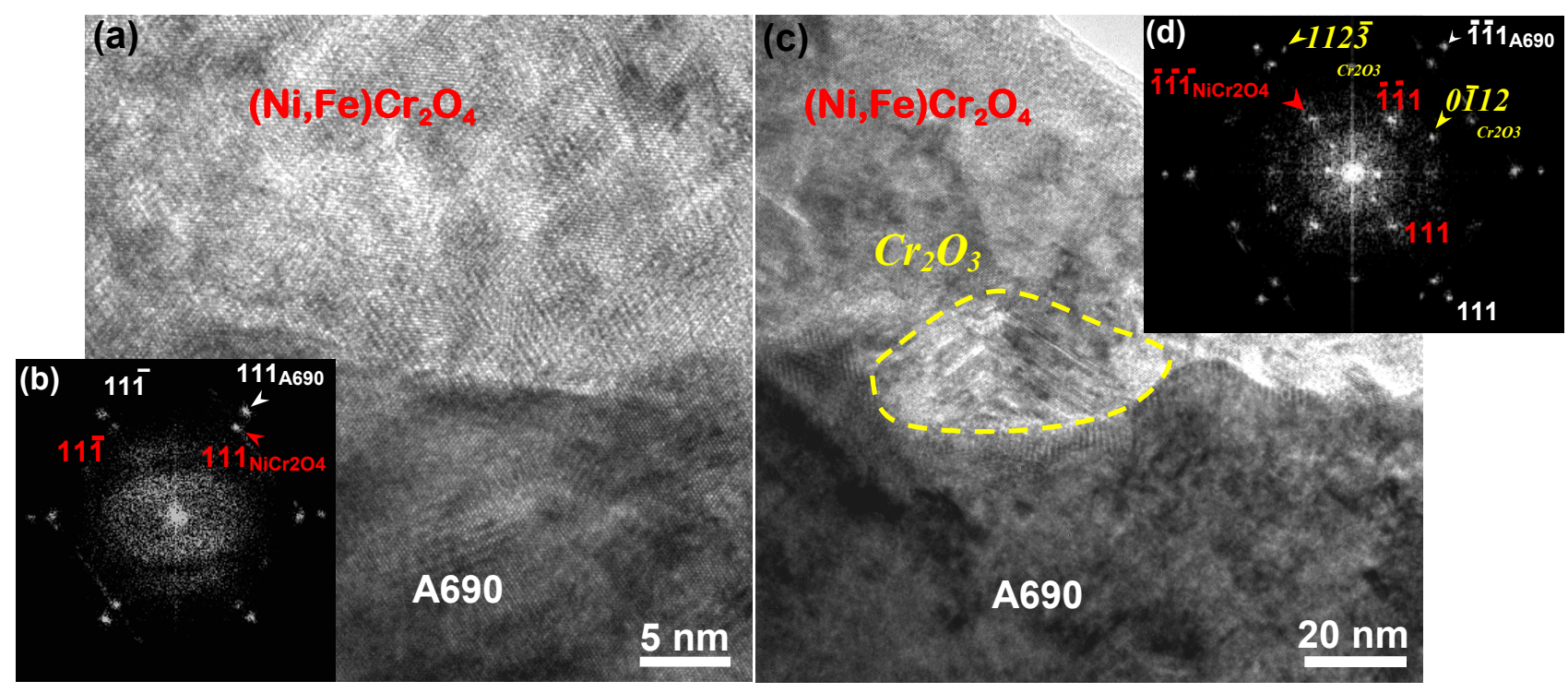

Fig. 4. HRTEM image ((a), (c)) and associated Fourier transform diffractogram ((b), (d)) of the oxide-alloy interface for Alloy 690 corroded for $858 \mathrm{~h}$.

Concerning the external layer, HRTEM observations combined with FT diffractogram analysis (Fig. 5) identified the large crystallites as having of $\mathrm{NiFe}_{2} \mathrm{O}_{4}$-structure with partial substitution of $\mathrm{Fe}$ atoms by $\mathrm{Cr}$ as can be deduced from EDX compositional profiles. FT indexing also established the existence of an orientation relationship with respect to the internal layer of the type:

$$
(111)_{\mathrm{NiFe}_{2} \mathrm{O}_{4}} / /(101)_{\mathrm{NiCr}_{2} \mathrm{O}_{4}} \text { and }[110]_{\mathrm{NiFe}_{2} \mathrm{O}_{4}} / /[010]_{\mathrm{NiCr}_{2} \mathrm{O}_{4}} \text {. }
$$

From these observations, a duplex structure of the oxide layer is clearly established. It consists on large scattered crystallites rich in $\mathrm{Fe}$ and having a $\mathrm{NiFe}_{2} \mathrm{O}_{4}$ structure forming the external layer, and a subjacent continuous and compact $\mathrm{Cr}$-rich layer with a $\mathrm{NiCr}_{2} \mathrm{O}_{4}$ crystallographic structure. Nanometer-sized nodules of $\mathrm{Cr}_{2} \mathrm{O}_{3}$-structure were also present along the oxide-matrix interface.
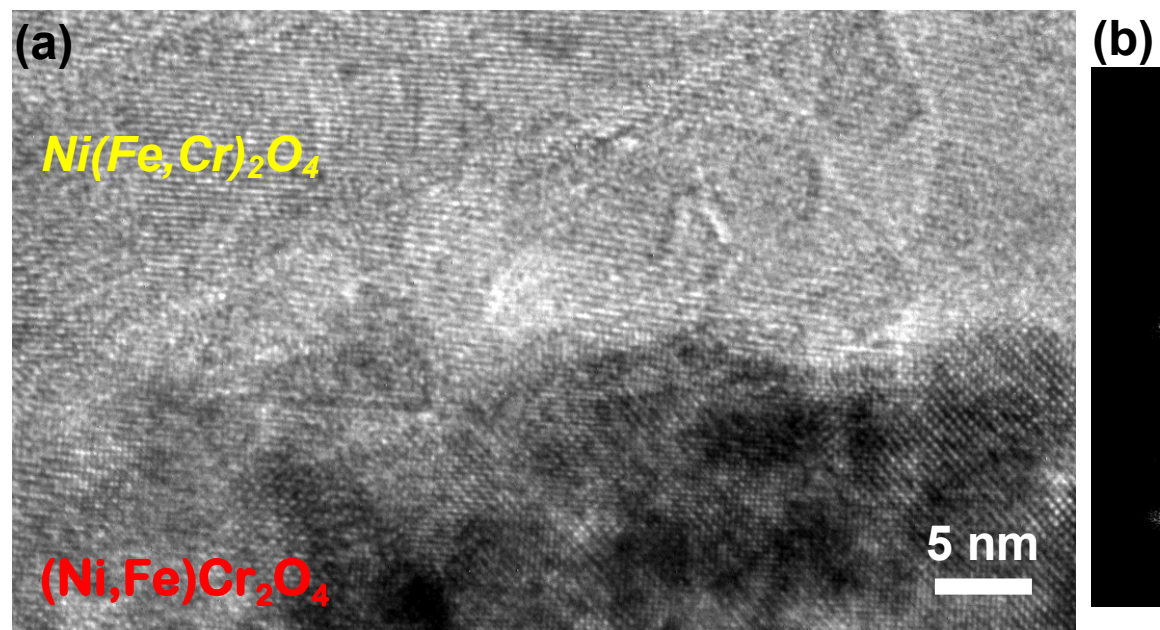

Fig. 5. HRTEM image (a) and associated Fourier transform diffractogram (b) of the internal oxideexternal oxide for Alloy 690 corroded for $858 \mathrm{~h}$.

Influence of surface defects. Samples implanted with xenon and corroded during 304 hours in PWR conditions were analyzed by TEM techniques. HRTEM observations shown on Fig. 6(a), revealed the same structure for the internal oxide layer with a continuous layer composed of 
$\mathrm{NiCr}_{2} \mathrm{O}_{4}$ spinel structure, and $\mathrm{Cr}_{2} \mathrm{O}_{3}$ nodules at the oxide scale/alloy interface. These phases were identified by using Fourier transform diffractogram (Fig. 6(b)). What must be underlined is that the number of $\mathrm{Cr}_{2} \mathrm{O}_{3}$ nodules was greater for the sample which had been implanted.

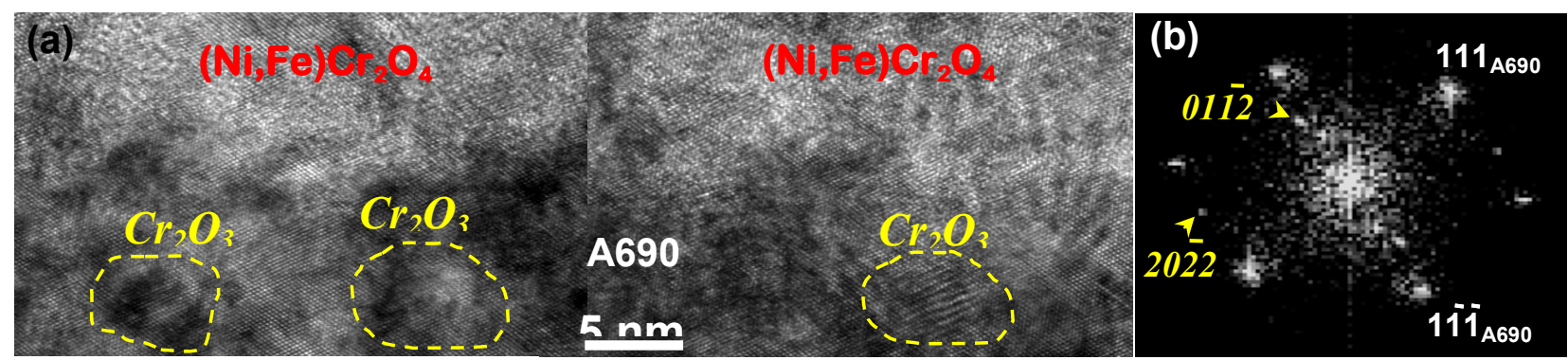

Fig. 6. HRTEM image (a) and Fourier transform diffractogram associated with the circles (b) of the alloy-internal oxide for Alloy 690 implanted with xenon and corroded for 304h.

In Fig. 7, comparison of the internal oxide layer between an implanted sample corroded for $304 \mathrm{~h}$ (Fig. 7(a)) and a non implanted sample and corroded for 858 h (Fig. 7(b)) was made by using TEM image in the under-focused mode. Two main differences were observed. On one hand, the oxide structure highlighted a higher density of grains or sub-grains for the oxide formed on the implanted alloy. The grain size was about $20 \mathrm{~nm}$ for the oxide formed on the non implanted sample whereas it was of few $\mathrm{nm}$ for the xenon-implanted sample. It must also be underlined that the oxide scale was thicker on the xenon-implanted sample than on the non-implanted sample although it was corroded for a shorter time.
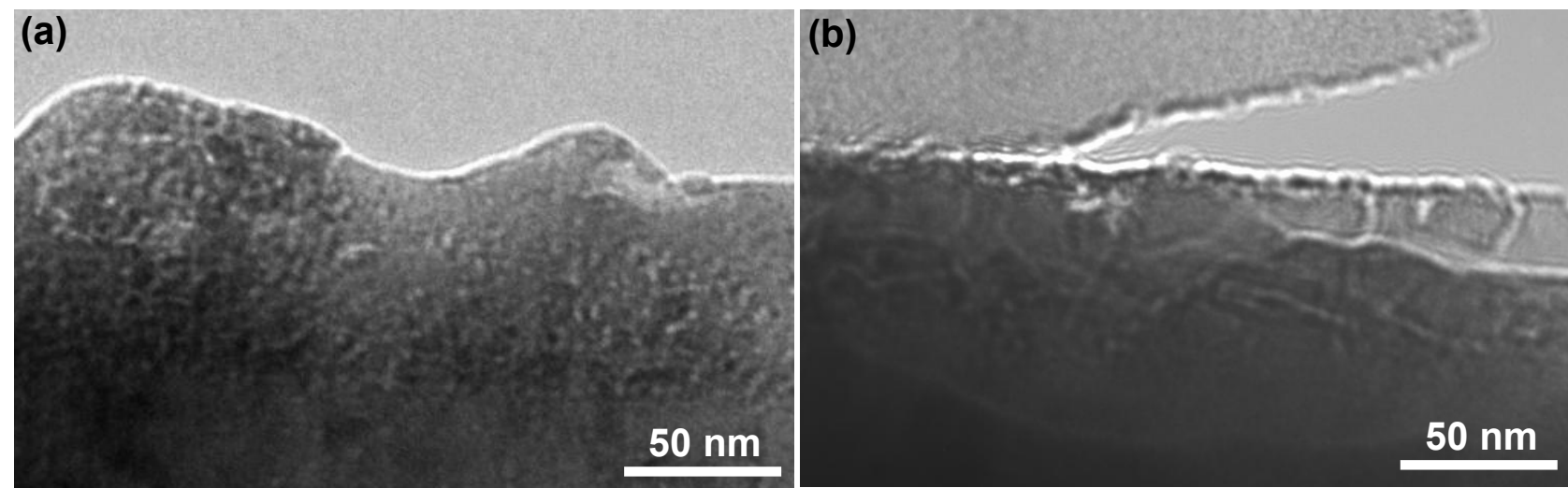

Fig. 7. TEM comparison (under-focused mode) of the continuous oxide layer of (a) Alloy 690 implanted with Xenon and corroded for $304 \mathrm{~h}$, and (b) Alloy 690 corroded for $858 \mathrm{~h}$.

\section{Discussion}

The TEM observations performed on samples corroded for different durations enabled the proposal of a global structure of the oxide layer formed on nickel based alloys in PWR primary conditions (Fig. 8). This oxide layer is divided in two parts. The internal layer is composed of two different oxides: a continuous scale whose composition is close to $(\mathrm{Ni}, \mathrm{Fe}) \mathrm{Cr}_{2} \mathrm{O}_{4}$ with nodules of $\mathrm{Cr}_{2} \mathrm{O}_{3}$ present at the oxide scale/alloy interface between the alloy and the continuous spinel. The external layer consists of scattered octahedral crystallites of a spinel oxide whose composition is close to $\mathrm{NiFe}_{2} \mathrm{O}_{4}$. 


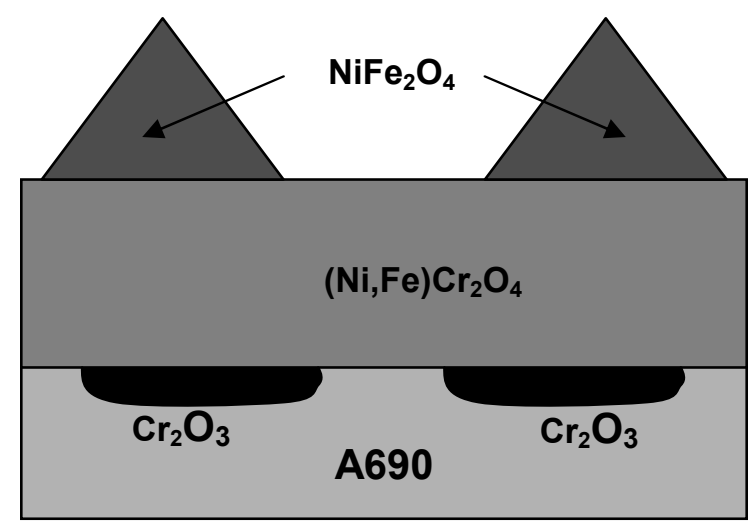

Fig. 8. Schematic diagram of the oxide layers formed on nickel base alloy in PWR primary conditions.

These crystallites rich in iron have also been observed on sample of Ni-30Cr ( an alloy without iron) which indicates that they are due to precipitation phenomenon as proposed by different authors $[3,6]$. The TEM observations of a sample with a defective surface created by xenon implantation provided indications of the mechanism of oxide formation. For the continuous chromite layer, an increase of the surface defect density induced a decrease of the oxide grain size. These surface defects also resulted in a thicker oxide. These two results well agree with a growth mechanisms controlled by a diffusion step at the oxide grain boundaries. Concerning the $\mathrm{Cr}_{2} \mathrm{O}_{3}$ nodules, a nucleation and growth mechanism can be considered for the formation of this phase. The defects at the alloy/oxide scale interface play the role of preferential sites for the nodule nucleation, increasing the rate of this process $[14,15]$. Thus, the sample with a more highly-defective surface develops more nodules. Contrary to some literature results, no depleted chromium layer in the alloy was observed in this study. Such a depleted layer could be attributed to the establishment of a continuous layer of $\mathrm{Cr}_{2} \mathrm{O}_{3}$ when the defective alloy surface state results in a sufficiently high nucleation frequency [16] for the formation of this phase. Once this layer is continuous, the corrosion mechanism can change and a chromium depletion can appear in the alloy, resulting from the preferential oxidation of chromium.

\section{Conclusions}

The multi layer oxide structure formed during corrosion of nickel base alloys in PWR primary coolant has been characterized by TEM. The internal layer is divided into a continuous film of $(\mathrm{Ni}, \mathrm{Fe}) \mathrm{Cr}_{2} \mathrm{O}_{4}$ and nodules of $\mathrm{Cr}_{2} \mathrm{O}_{3}$ at the alloy oxide interface. The external layer also is composed of a spinel oxide rich in iron. Moreover the role of the near surface alloy defects has been studied showing that it changes the crystallinity of the continuous spinel scale (which modifies the diffusion processes at the grain boundaries) and the nucleation frequency of $\mathrm{Cr}_{2} \mathrm{O}_{3}$ (which could entail the formation of a continuous layer of this phase).

\section{Acknowledgements}

The authors would like to thank Michel DROUET from the Physical Metallurgy Laboratory of the University of Poitiers for the xenon implantation. 


\section{References}

[1] N.A. McIntyre, D. G. Zetaruk and D. Oven: J. Eletrochem. Soc. Vol. 126 (1979), p.750.

[2] L. Guinard, O. Kerrec, D. Noel, S. Gardey and F. Coulet: Nuclear Energy. Vol. 36 (1997), p. 19

[3] F. Carette, M.C. Lafont, G. Chataignier, L. Guinard and B. Pieraggi: Surf. Interf. Anal. Vol. 34 (2002), p. 135.

[4] F. Carette, L. Guinard, B. Pieraggi, in: Proceedings of the International Conference on water Chemistry of Nuclear Reactor Systems, Operation Optimisation and New Developments, (2002).

[5] A. Machet, A. Galtayries, P. Marcus, P. Combrade, P. Jolivet and P. Scott: Surf. Interf. Anal. Vol. 34 (2002), p. 197.

[6] F. Carette, M.C. Lafont, L. Legras, L. Guinard and B. Pieraggi: Mat. High. Temp. Vol. 20 (2003), p. 581.

[7] A. Machet, A. Galtayries, S. Zanna, L. Klein, V. Maurice, P. Jolivet, M. Foucault, P. Combrade, P. Scott and P. Marcus: Electrochimica Acta. Vol. 49 (2004), p. 3957.

[8] S. Gardey: PhD Thesis, Univ. of Paris 6 (1998).

[9] F. Carette, PhD Thesis, Institut National Polytechnique de Toulouse (2002).

[10] A. Machet, PhD Thesis, Univ. of Paris 6 (2004).

[11] F. Delabrouille, PhD Thesis, Institut National Polytechnique de Toulouse (2004).

[12] J. Panter, PhD Thesis, Institut National Polytechnique de Toulouse (2002).

[13] L. Guinard, O. Kerrec, S. Gardey, D. Noel, in: Proceeding of international conference on water chemistry in nuclear power plants, Niigata (Japan), (1998).

[14] B. Delmon, in: Introduction à la Cinétique Hétérogène, Technip, Paris (France), (1969).

[15] L. Favergeon, PhD Thesis, Ecole Nationale Supérieure des Mines de Saint Etienne, (2006).

[16] M. Soustelle, M. Pijolat: Solid state ionics, Diffusion and reactions Vol. 95 (1997), p. 33. 